\title{
Inducible regulation of GDNF expression in human neural stem cells
}

\author{
WANG ShuYan, REN Ping, GUAN YunQian, ZOU ChunLin, \\ FU LinLin \& ZHANG Yu* \\ Cell Biology Room, Xuanwu Hospital, Capital Medical University, and Key Laboratory of Neurodegeneration, \\ Ministry of Education, Beijing 100053, China
}

\begin{abstract}
Glial cell derived neurotrophic factor (GDNF) holds promises for treating neurodegenerative diseases such as Parkinson's disease. Human neural stem cells (hNSCs) have proved to be a suitable cell delivery vehicle for the safe and efficient introduction of GDNF into the brain. In this study, we used hNSCs-infected with a lentivirus encoding GDNF and the hygromycin resistance gene as such vehicles. A modified tetracycline operator 7 (tetO7) was inserted into a region upstream of the EF1- $\alpha$ promoter to drive GDNF expression. After hygromycin selection, hNSCs were infected with a lentivirus encoding a KRAB-tetracycline repressor fusion protein (TTS). TTS bound to tetO7 and suppressed the expression of GDNF in hNSCs. Upon administration of doxycycline (Dox) the TTS-tetO7 complex separated and the expression of GDNF resumed. The hNSCs infected with GDNF expressed the neural stem cell specific markers, nestin and sox2, and exhibited no significant change in proliferation rate. However, the rate of apoptosis in hNSCs expressing GDNF was lower compared with normal NSCs in response to actinomycin treatment. Furthermore, a higher percentage of Tuj-1 positive cells were obtained from GDNF-producing NSCs under conditions that induced differentiation compared to control NSCs. The inducible expression of GDNF in hNSCs may provide a system for the controllable delivery of GDNF in patients with neurodegenerative diseases.
\end{abstract}

neural stem cells, GDNF, lentivirus vectors, tetracycline operator

Citation: Wang S Y, Ren P, Guan Y Q, et al. Inducible regulation of GDNF expression in human neural stem cells. Sci China Life Sci, 2013, 56: 32-39, doi: $10.1007 / \mathrm{s} 11427-012-4424-6$

Glial cell line-derived neurotrophic factor (GDNF) is a potent and specific neurotrophic factor for dopaminergic neurons [1]. Expression of GDNF in the striatum has been shown to prevent neurodegeneration and promote sprouting of nigral dopaminergic neurons in animal models and clinical studies of Parkinson's disease (PD) [2,3]. However, attempts to apply GDNF clinically have so far been disappointing because of its poor penetration across the bloodbrain-barrier. Of several GDNF delivery methods, cellbased delivery systems provide a promising approach for treating PD. Human neural stem cells exhibit the potential

*Corresponding author (email: yaz@bjsap.org) to differentiate into neurons, astrocytes and oligodendrocytes[4,5], and may therefore provide an additional benefits for repair and replacement [6]. Several ex-vivo gene therapy studies have shown that GDNF-modified hNSCs could survive, migrate, secrete GDNF and differentiate into various cell types at the transplantation site in animal models of PD and amyotrophic lateral sclerosis (ALS) [7-9]. These studies suggest that GDNF-expressing hNSCs can provide a potential therapeutic strategy for treating neurodegenerative diseases.

Although GDNF has the potential to improve the survival of dopaminergic neurons, continuous expression of GDNF leads to side effects such as aberrant axonal sprout- 
ing and down-regulation of tyrosine hydroxylase [10]. Therefore, it is necessary to control the level of GDNF production at the site of cell transplantation in the brain. Transplanted cells modified with the GDNF gene are the ideal choice for controlling GDNF protein expression in the brain. To regulate foreign gene expression, the tetracycline-on (tet-on) [11] and tetracycline-off (tet-off) [12] operator systems have been developed. In the tet-off system, the continued presence of doxycycline (Dox) is required to suppress the expression of the gene of interest and therefore is not ideal where over-dosing of GDNF is a concern. However, one disadvantage of the "tet-on" system is the leakage problem, whereby expression of the target gene is not completely shut off in the absence of Dox [13]. To precisely and reversibly control the expression GDNF, we applied an improved inducible lentiviral system comprising a modified tetracycline operator 7 (tetO7) and a tetracycline repressor fusion protein (TTS) modified by Betty Zhou [14]. In this system, one lentiviral vector expresses TTS and RFP, and the second vector expresses GDNF (or GFP) and the hygromycin B resistance gene. When cells are co-infected with these two vectors, TTS can bind to tetO7 and suppress GDNF (or GFP) expression. Dox administration releases TTS from tetO7 to reversibly induce GDNF (or GFP) expression. This system can tightly control the expression of a target gene and was thus adopted in the present study. In this study, we investigated whether GDNF expression could be precisely and reversibly controlled in hNSCs infected with two inducible lentiviral vectors.

\section{Materials and methods}

\subsection{Culture of human fetal neural stem cells}

Neural stem cells were prepared from the forebrain cortex of aborted embryos (16 weeks post conception). Briefly, fetal tissues were treated with $0.05 \%$ trypsin-EDTA (Invitrogen, Carlsbad, CA, USA) for 15 minutes at $37^{\circ} \mathrm{C}$, following which trypsin inhibitor (Sigma-Aldrich, St. Louis, MO, USA) was added to terminate the enzymatic reaction. DNase (Sigma-Aldrich) was added to eliminate DNA released from the tissues. Single cell suspensions were harvested and filtered through $400-\mu \mathrm{m}$ meshes to eliminate cell clusters. The single cell suspensions were plated onto cell culture dishes at a density of $2 \times 10^{5}$ cells $\mathrm{mL}^{-1}$. The neurospheres that formed after 5-7 days in culture were propagated in growth medium containing DMEM-F12 (Invitrogen), N2 (Invitrogen), $20 \mathrm{ng} \mathrm{mL}{ }^{-1}$ EGF (R\&D systems,

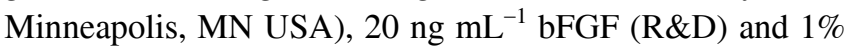
BSA (Invitrogen). Half of the medium was changed every three days. For neuronal differentiation, the hNSCs were plated onto poly-D-lysine/laminin coated plates in differentiation medium comprising DMEM-F12, N2, B27 and 1\% BSA for two weeks.

\subsection{Lentiviral production and infection}

The lentiviral vector was constructed on the basis of a previously published vector, DUET101 [14]. We replaced the GFP sequence with the GDNF sequence. A tetO7 operator was also inserted upstream of the EF-1 $\alpha$ promoter. Lentiviral vectors encoding TTS and red fluorescent protein (RFP) were also constructed. The lentiviruses were produced by transient transfection. $293 \mathrm{~T}$ cells were plated at $6 \times 10^{6}$ cells per $100 \mathrm{~cm}$ poly-D-lysine(Sigma-Aldrich) coated dish and incubated overnight. Three lentiviral vector plasmids $(6 \mu \mathrm{g}$ of DUET101-GDNF, DUET101-GFP or TTS, $2 \mu \mathrm{g}$ of PMD.G, and $8 \mu \mathrm{g}$ CMV8.91) were mixed with Lipofectamine 2000 (Invitrogen) according to the manufacturer's instructions and added to the 293T cells. After incubating for 8 hours, the medium was replaced with fresh medium comprising DMEM/F12 (Invitrogen) and ITS supplement (Invitrogen). After $48 \mathrm{~h}$, the viral supernatants were collected and concentrated by ultra-centrifugation. Viral titers were determined before hNSC infection. 293T cells were plated at a density of $1 \times 10^{5}$ cells per well in six-well plates and cultured overnight. Concentrated DUET101-GFP/TTS viral supernatants and polybrene $\left(8 \mu \mathrm{g} \mathrm{mL}^{-1}\right.$ Sigma) were added to the prepared $293 \mathrm{~T}$ cells. $48 \mathrm{~h}$ later, the percentages of GFP and RFP positive cells were counted by FACS. Viral titers were calculated as transfection units (TU). If $10 \%$ of the cells were GFP positive following infection with 0.02 $\mathrm{mL}$ of viral supernatant, then the viral titer is: $10 \% \times \operatorname{target}$ cell number $\left(2 \times 10^{5}\right.$ for $293 \mathrm{~T}$ cells $) / 0.02 \mathrm{~mL}=1 \times 10^{6} \mathrm{TU}$ $\mathrm{mL}^{-1}$, assuming that the $293 \mathrm{~T}$ cells doubled when infected.

Prior to infecting the hNSCs, neurospheres were dissociated into single cells with accutase and seeded at a density of $2 \times 10^{5}$ cells $\mathrm{cm}^{-2}$ in six-well plates. After incubating for $72 \mathrm{~h}$, concentrated viral supernatants for DUET101-GDNF or DUET101-GFP (control) were added to the NSC media together with $4 \mu \mathrm{g} \mathrm{mL}^{-1}$ polybrene. The multiplicity of infection (MOI) was determined as the ratio of transfection units to cell number. Twenty-four hours later, the infection medium was aspirated and replaced with fresh NSC medium. After expanding the infected hNSCs for two passages, $100 \mu \mathrm{g} \mathrm{mL}^{-1}$ hygromycin was added to enrich the successfully infected cells. For regulation of GDNF expression, the selected hNSCs were infected with lentiviruses encoding TTS and RFP. The expression level of GDNF in hNSCs co-infected with DUET101-GDNF and TTS viral supernatants was determined by ELISA.

\subsection{ELISA}

Equal numbers of hNSCs infected with DUET101-GDNF were seeded at an appropriate density and infected with different viral MOIs, following which the medium was collected 24 and $48 \mathrm{~h}$ later for ELISA assay. To determine whether the hNSCs secreted GDNF continuously and stably, the medium was collected at different time points (days 3, 7, 14 and 21) and analyzed by ELISA. To determine whether 
GDNF secretion by hNSCs could be regulated or controlled, the medium from the hNSCs was collected on days 1, 3 and 5 after introducing the TTS virus. Then, different doses of Dox $\left(2,5,10,20,50 \mathrm{ng} \mathrm{mL}^{-1}\right)$ were added and the medium was collected on days 1, 3, 5, 7 and 9 after Dox administration. The concentration of GDNF in the medium was determined using an ELISA kit (Promega Corporation, Madison, Wisconsin, USA) according to the manufacturer's instructions.

\subsection{Real-time PCR}

Total RNA was purified from hNSCs with TRIzol regent (Invitrogen) and treated with RNase-free DNase (Promega) to remove genomic DNA contamination. Two micrograms of RNA were used in a reverse transcription reaction with random primers (Invitrogen) and Superscript II reverse transcriptase kit (Invitrogen) according to the manufacturer's protocol. For quantification of GDNF mRNA expression, human 18s RNA was used as the internal reference. Real-time PCR was performed using the SYBR Green Premix Taq kit (Takara Biotechnology Co., Ltd, Dalian, China). Relative mRNA levels were quantified by the comparative $\mathrm{Ct}$ method. Primer sequences are listed in Table 1.

\subsection{Immunohistochemistry}

Differentiated hNSCs were fixed with PBS containing 4\% paraformaldehyde for $10 \mathrm{~min}$ at room temperature. After washing with PBS, the cells were treated with PBS containing 3\% normal donkey serum (Jackson ImmunoResearch Laboratories, Inc., Baltimore, USA) and $0.1 \%$ Triton X-100 for $60 \mathrm{~min}$ at room temperature. Primary antibodies for nestin $(1: 400$, Merck Millipore Headquarters, Billerica, USA), Tuj-1 (1:200, Millipore) and GFAP ( $1: 200$, Millipore) were added and incubated overnight at $4{ }^{\circ} \mathrm{C}$. The secondary antibodies used were cyanine 3 (Cy3)-conjugated donkey anti-mouse $\operatorname{IgG}$ (1:500, Jackson) and cyanine 2 (Cy2)-conjugated donkey anti-mouse IgGs. Nuclei were stained with DAPI.

\subsection{Apoptosis assays}

Normal hNSCs and LV-GDNF NSCs were exposed to actinomycin-D $\left(1 \mu \mathrm{mol} \mathrm{L}{ }^{-1}\right)$ for $12 \mathrm{~h}$. Apoptosis was evaluated as the percentage of annexin V-positive cells using an apoptosis kit (Invitrogen) according to the manufacturer's instructions.

\section{Results}

\subsection{The expression level of GDNF in hNSCs infected with DUET101-GDNF virus increased significantly in a manner proportional to the MOI value}

We constructed lentiviral vectors encoding GDNF under the control of the EF-1 $\alpha$ promoter and the tet-O7 operator (Figure 1A). The modified hNSCs were resistant to hygromycin and thus could be enriched. We first determined the infection efficiency of DUET101-GFP as a control for DUET101-GDNF viral infection. The average titer of concentrated DUET101-GFP viral supernatants was about $5 \times 10^{7} \mathrm{TU} \mathrm{mL}^{-1}$. hNSCs infected with DUET101-GFP virus at different MOI values $(0,0.5,1,2.5,5,10)$ were analyzed by FACS. The results indicated that $44.5 \%$ of hNSCs were GFP positive when MOI=1 (Figure 1E). The percentage of GFP positive cells increased gradually with the MOI value. To purify successfully infected cells, hygromycin was added to the culture medium. After hygromycin selection, the percentage of GFP-positive cells increased to $99.5 \%$ (Figure $1 F)$. The same selection procedure was performed after hNSCs were infected with DUET101-GDNF. We determined the GDNF expression level in the supernatants of purified hNSC infected with DUET101-GDNF at different MOI values $(0,0.5,1,2.5,5,10)$ by ELISA. The results indicated that the GDNF expression level in hNSCs infected with DUET101-GDNF increased significantly compared with control cells $(\mathrm{MOI}=0)$. Furthermore, the GDNF expression level increased proportional to the MOI value (Figure 2A). At the same time, the GNDF mRNA levels in hNSCs infected with DUET101 GDNF at different MOI values $(0,0.5,1,2.5,5,10)$ were also analyzed by real-time PCR. The results indicated that the GDNF mRNA levels in infected hNSCs increased proportional to the MOI value (Figure 2B). We further determined the GDNF expression level at different time points (days 3, 7, 14, 21 40) after infection. The results showed that infected hNSCs could secrete GDNF continuously and stably. The GDNF expression level in infected hNSCs was maintained at a higher level than in control cells (Figure 2C). These results indicated that GDNF secretion was significantly improved in hNSCs infected with DUET101-GDNF. Furthermore, the GDNF expression level in hNSCs increased with the MOI value. The hygromycin-selected hNSCs over-expressed GDNF continuously and stably. These data indicate that GDNF expression can be controlled in human fetal neural stem cells.

\subsection{Tight regulation of GNDF expression in hNSCs}

On the basis that purified hNSCs over-expressing GDNF could be cultured and propagated, the TTS vector was introduced to regulate and control GDNF expression. The TTS vector encodes a fusion protein containing a tetracycline repressor (tetR) domain and RFP (Figure 1B). The hNSCs successfully infected with TTS were RFP positive (Figure 3A and B). When these neurospheres were dissociated into single cells, FACS analysis indicated that about $96.65 \%$ of the cells were RFP positive (Figure 3C and D). These results indicated that DUET101-GDNF and TTS were both expressed in hNSCs. Therefore, the tetR fusion 

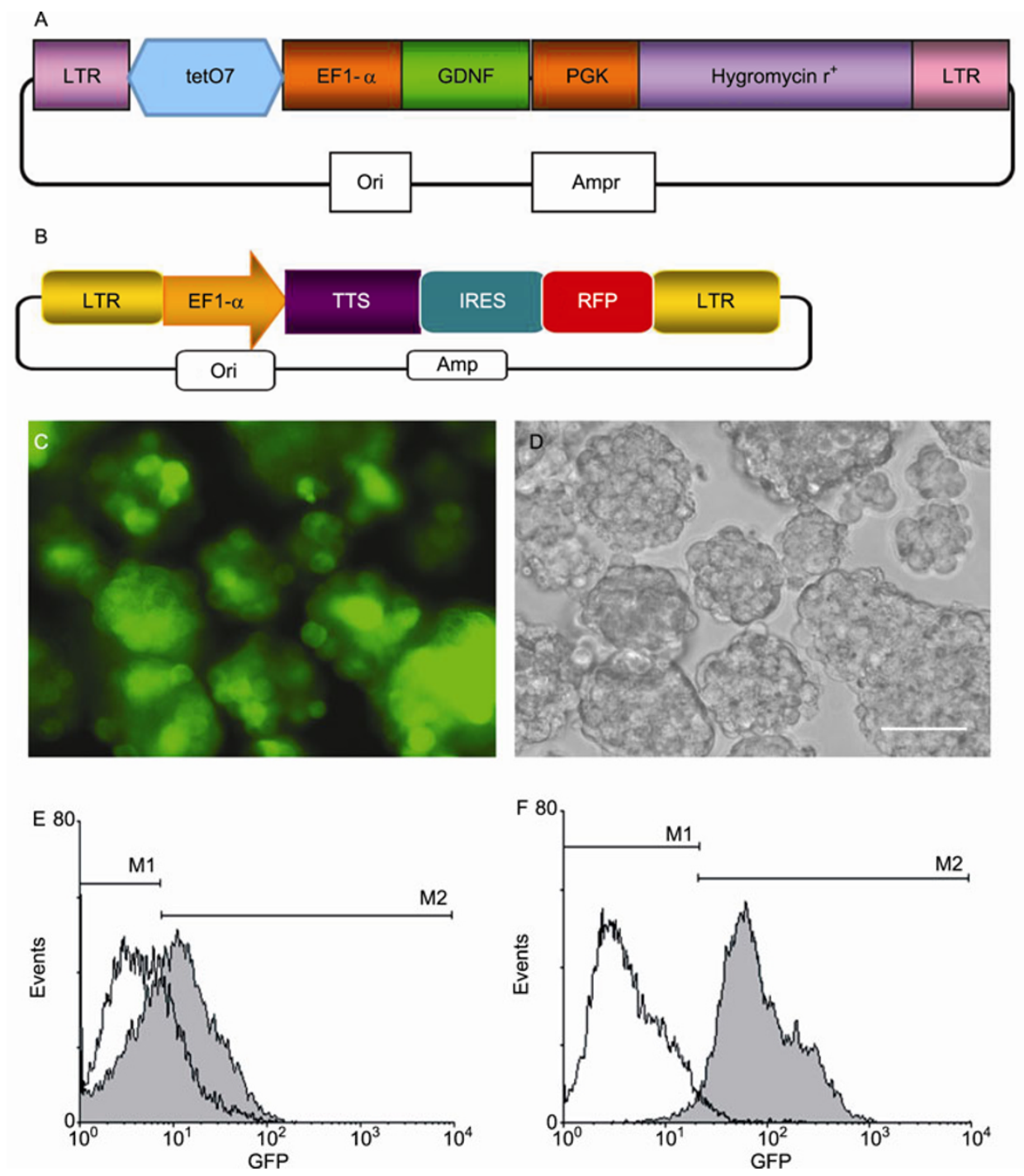

Figure 1 Schematic representation of the GDNF inducible lentiviral vectors and the infection efficiency assay. A, The DUET101-GDNF lentiviral vector expresses the GDNF and hygromycin resistance genes driven by EF1-a and PGK, respectively. A modified tetracycline operator 7 (tetO7) was inserted upstream of EF1- $\alpha$. B, The TTS vector encodes the TTS protein (a fusion protein of KRAB and tetracycline repressor) and red fluorescent protein (RFP). C, Bright field view of neurospheres infected with DUET101-GFP. D, Fluorescence micrograph showing GFP-positive neurospheres. Scale bar, $50 \mu \mathrm{m}$. E, Prior to hygromycin selection, about $44.5 \%$ of cells were GFP-positive. F, Following hygromycin selection, about $99.5 \%$ of cells were GFP-positive.

protein can bind to the tetracycline operator, tetO7, and suppress gene expression in the absence of Dox. We collected the medium from the hNSCs on days 1, 3 and 5 after infection with the TTS virus. The concentration of secreted GDNF was analyzed by ELISA. The results showed that extracellular GDNF secretion declined to background levels after introduction of the TTS vector (Figure 3F). The decreased GDNF secretion indicated that GDNF expression could be inhibited by introducing the TTS vector. Following Dox treatment, GDNF expression was restored by release of TTS from tetO7. Following this confirmation that GDNF expression in hNSCs could be suppressed with TTS, different doses of Dox $\left(2,5,10,20\right.$ and $\left.50 \mathrm{ng} \mathrm{mL}^{-1}\right)$ were added and the medium was collected for GDNF ELISA on days 1, 3, 5, 7 and 9. The results indicated that the concentration of secreted GDNF increased gradually up to day 7 after addition of Dox ( $\geqslant 5 \mathrm{ng} \mathrm{mL}^{-1}$ ) (Figure 3G). In hNSCs co-infected with DUET101-GFP and TTS viruses, a gradual transition from RFP to GFP expression was seen (Figure 3E). Upon removal of Dox from the medium, the expression of GDNF was rapidly shut off (data not shown). The up- and down-regulation of GDNF can be repeated for several cycles by the addition and removal of Dox. The response to Dox treatment was quite sensitive and a dose of Dox as low as $5 \mathrm{ng} \mathrm{mL}^{-1}$ was sufficient to drive the expression of GDNF. These results suggest that GDNF can be tightly and efficiently con- 

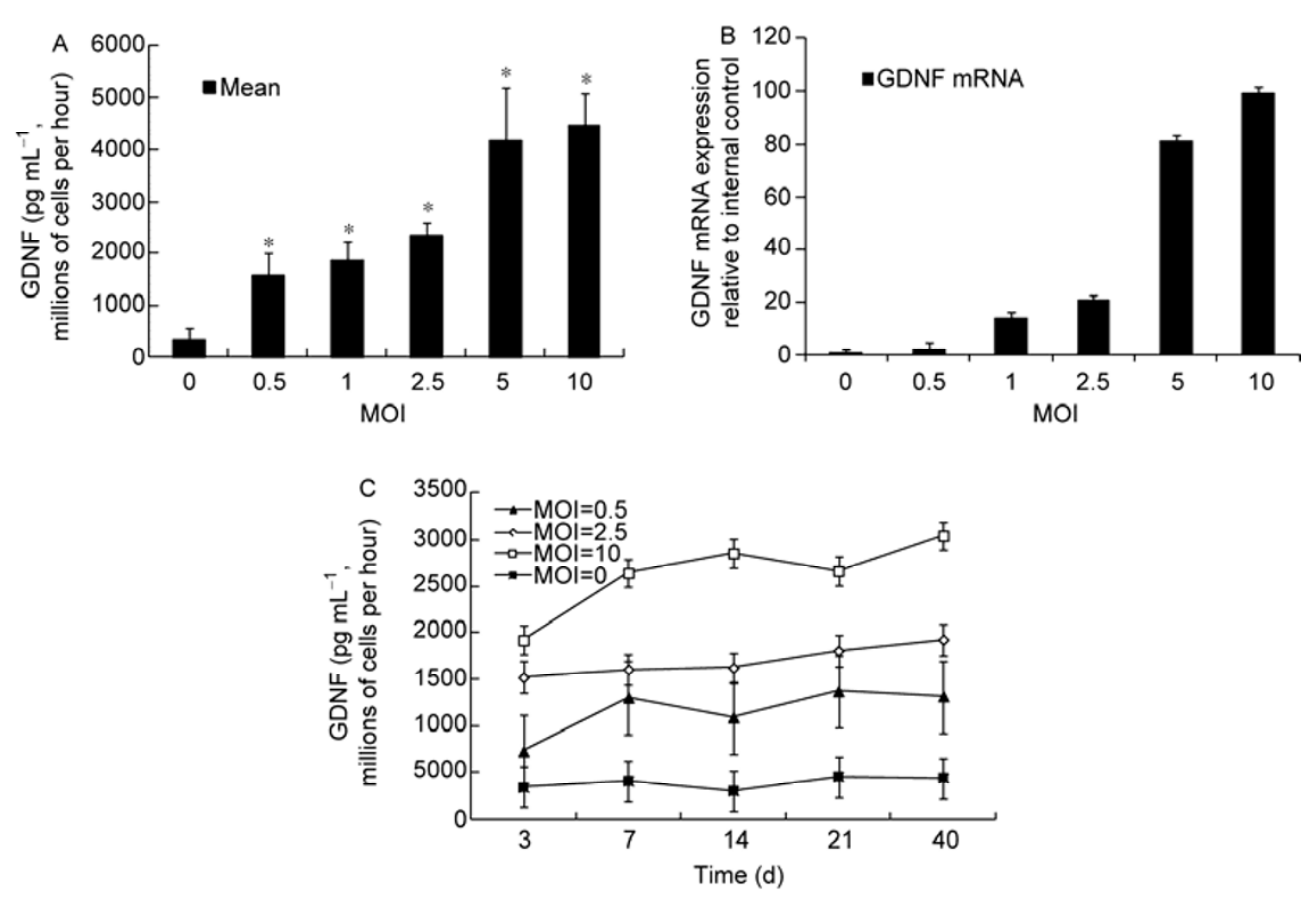

Figure 2 The GDNF expression level in hNSCs infected with DUET101-GDNF virus increased significantly and was directly proportional to MOI value. A, ELISA analysis of the concentration of secreted GDNF at different MOI values. Compared with control, the concentration of GDNF secreted by hNSCs infected with DUET101-GDNF increased significantly and was proportional to the MOI value (ANOVA analysis; *, $P<0.01$ ). B, Real-time PCR analysis of GDNF mRNA levels at the indicated MOI values. The GDNF mRNA expression level in hNSCs infected with DUET101-GDNF was significantly elevated and was proportional to the MOI value after normalization to an internal reference (human 18 s ribosome RNA) (ANOVA analysis; *, P<0.01). C, ELISA analysis of GDNF expression for $40 \mathrm{~d}$ after infection of hNSCs with different MOIs of GDNF lentivirus. DUET101-GDNF-infected hNSCs over-expressed GDNF stably and continuously, and the relationship between GDNF secretion and MOI was stable.

Table 1 Real-time PCR primer list

\begin{tabular}{ll}
\hline \multicolumn{1}{c}{ Primer name } & \multicolumn{1}{c}{ Primer sequence } \\
\hline GDNF forward & ACTGACTTGGGTCTGGGCTATG \\
GDNF reverse & TTTGTCACTCACCAGCCTTCTATTT \\
Human $18 \mathrm{~S}$ forward & ACTCAACACGGGAAACCTCA \\
Human $18 \mathrm{~S}$ reverse & AACCAGACAAATCGCTCCAC \\
\hline
\end{tabular}

trolled in our system.

\subsection{Decreased apoptosis and increased neuronal dif- ferentiation in hNSCs infected with DUET101-GDNF}

To investigate whether GDNF over-expression would affect the characteristics of hNSCs, we examined antigen expression, proliferation, apoptosis and differentiation in hNSCs infected with DUET101-GDNF and TTS. Immunohistochemistry demonstrated that hNSCs infected with DUET101-GDNF expressed the neural stem cell specific markers, nestin and sox2 (data not shown). When actinomycin-D was added to induce apoptosis, hNSCs infected with DUET101-GDNF showed a lower percentage of annexin V-positive cells compared with normal hNSCs (Figure 4A). After natural differentiation, the percentage of early neurons (Tuj-1 positive) and astrocytes (GFAP positive) was analyzed. The results showed that the percentage of Tuj-1 positive cells that differentiated from hNSCs infected with DUET101-GDNF increased significantly (Figure 4B). Comparison of the biological characteristics of infected and control cells indicated that antigen expression and proliferation were not changed by GDNF over-expression. Furthermore, apoptosis was reduced and neuronal differentiation was increased significantly in hNSCs over-expressing GDNF. These cellular characteristics may be considered desirable for the application of hNSCs to cell therapy for neurodegenerative disease.

\section{Discussion}

Certain potential adverse events, including extensive aberrant fiber sprouting downstream of striatal targets and lack of functional recovery, have been observed in studies using regular lentivirus-mediated GDNF delivery [10]. Although the mechanisms underlying these effects remain elusive, these results indicate that vector-mediated GDNF delivery may have to be maintained within a defined dose range to avoid over-dosing effects [15]. In this study, we demonstrated that an inducible lentiviral expression system can efficiently mediate the tight regulation of GDNF expression in human neural stem cells. The correlation between GDNF secretion and lentivirus MOI values provides the first layer of control over the desired expression of GDNF, i.e., by 

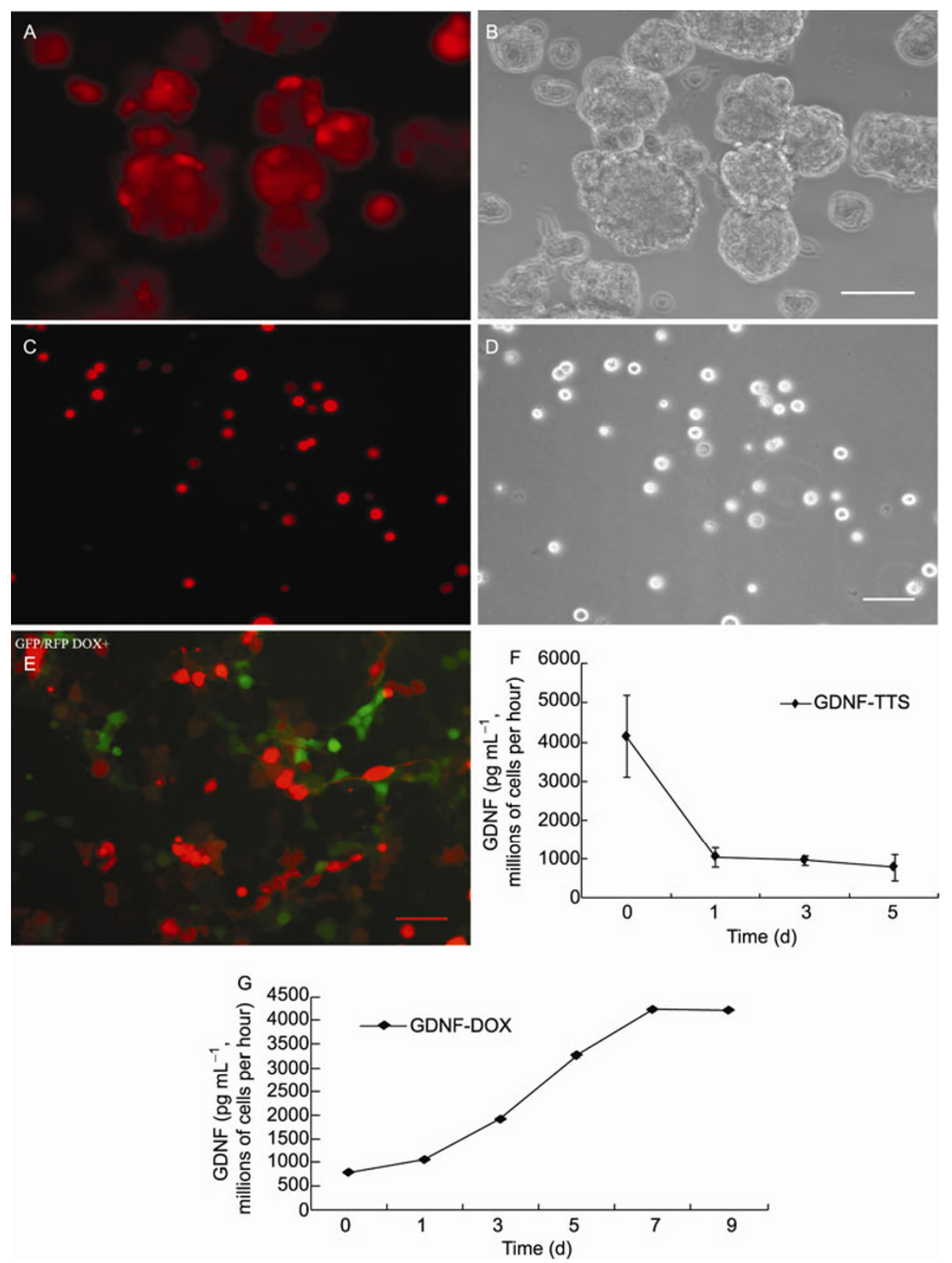

Figure 3 Tight regulation of GDNF secretion in hNSCs using an inducible lentiviral vector system. A, After infection with the TTS vector, neurospheres formed by DUET101-GDNF hNSCs were RFP positive under fluorescence microscopy. B, Bright field view of the neurospheres formed by LV-GDNF hNSCs shown in A. Scale bar, $50 \mu \mathrm{m}$. C, More than $90 \%$ of cells were RFP positive when the infected neurospheres were dissociated into single cells. D, Bright field view of the single cells dissociated from infected neurospheres shown in C. Scale bar, $100 \mu \mathrm{m}$. E, After Dox administration, the control group hNSCs co-infected with DUET101-GFP and TTS began to change from RFP to GFP positive. F, ELISA analysis of the concentration of GDNF secreted by DUET101-GDNF hNSCs infected with TTS (MOI=5). The concentration of GDNF secreted by DUET101-GNDF hNSCs decreased to the control level within $48 \mathrm{~h}$ after introduction of TTS. G, ELISA analysis of the concentration of GDNF secreted by hNSCs co-infected with DUET101-GDNF and TTS after Dox $\left(\geqslant 5 \mathrm{ng} \mathrm{mL}^{-1}\right)$ administration. The concentration of GDNF secreted by hNSCs co-infected with DUET101-GDNF and TTS increased gradually and returned to the unsuppressed level after treatment with Dox for 5-7 d.

adjusting the number of virus particles for infection of NSCs. The reversible Dox responsiveness suggests a convenient means to shut off GDNF expression when production of GDNF is no longer needed or has caused a side effect. The functionality of our inducible systems offers an opportunity for maintaining GDNF gene delivery within a defined dose range. We used KRAB-mediated repression and the tetracycline repressor, tetO7, in our inducible sys- tems. It has been demonstrated that KRAB-mediated repression can eliminate the leaky, non-specific expression that frequently affects transactivation-based conditional systems [16-18]. We demonstrated that GDNF expression in our system could reach a plateau level within three days, suggesting a fast response to Dox. If the Dox concentration was maintained at a certain level, GDNF secretion also remained stable. Upon removal of Dox, GDNF expression 

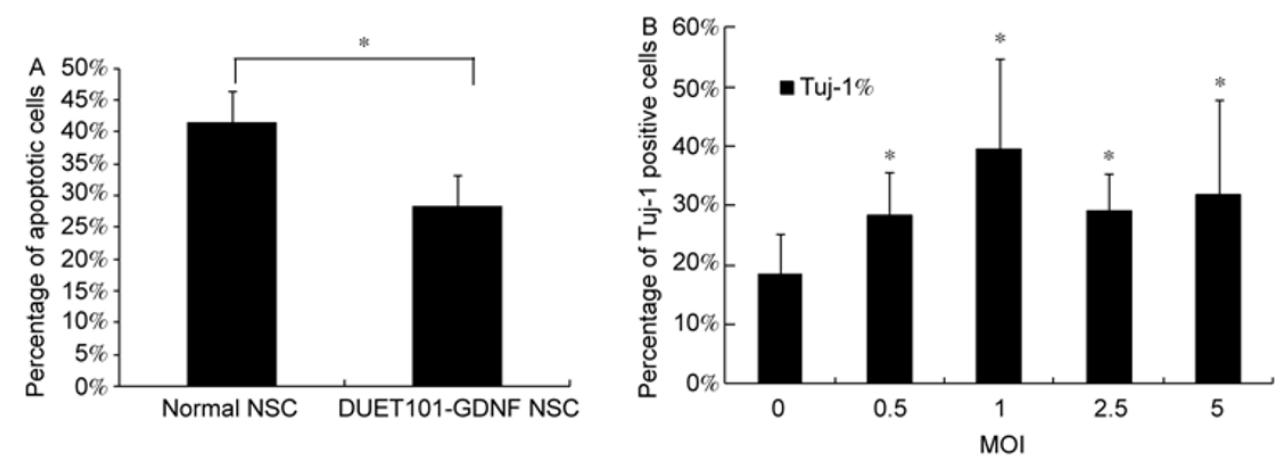

Figure 4 Apoptosis was reduced and neuronal differentiation was increased in hNSCs over-expressing GDNF. A, FACS analysis of annexin V-positive cells after treatment with actinomycin-D $\left(1 \mu \mathrm{mol} \mathrm{L} \mathrm{L}^{-1}\right)$. Annexin V-positive cells infected with DUET101-GDNF decreased by $13.2 \%$ compared with normal, non-infected hNSCs. *, $P<0.01$. B, Analysis of the percentage of Tuj-1 positive cells after natural differentiation of control and DUET101-GDNF hNSCs. The percentage of Tuj-1 positive LV-GDNF hNSCs increased significantly at different MOI values compared to normal hNSCs. *, $P<0.05$.

tapered off within 5-7 d. In the TTS-suppressed state, the GDNF protein levels did not show a significant difference compared with the control group (non-infected NSCs), suggesting that the leakage problem, which is a key concern for viral vector-based clinical approaches [19], does not exist in our system.

Because lentiviral vectors are retrovirus-based, the sequence of the vector itself and the foreign gene would integrate into the genomic DNA of the host cells. Therefore, the use of lentiviral vectors as gene therapy vehicles is controversial. We demonstrated that hNSCs did not show obvious changes in proliferation and antigen expression, indicating that the current system is relatively safe. Prior studies applying regular lentivirus-based cell therapy in animal models did not report any detectable cellular pathology or immune reactions [20], further suggesting that lentiviral vectors may be safe vehicles for GDNF delivery. Furthermore, compared with the control group, hNSCs infected with DUET101-GDNF showed a lower rate of apoptosis when treated with actinomycin and a higher percentage of Tuj-1 positive cells when cultured under conditions that induced differentiation. These results suggest that GDNF may not only exert a beneficial effect on injured neurons in the host but also promote the survival and neuronal differentiation of grafted hNSCs.

This work was supported by the National Basic Research Program of China (2007CB947704) and the High-level Technical Personnel Training of Health Plan of Beijing.

1 Lin L F, Doherty D H, Lile J D, et al. GDNF: a glial cell line-derived neurotrophicn factor midbrain dopaminergic neurons. Science, 1993, 260: 1130-1132

2 Kordower J H, Emborg M E, Bloch J, et al. Neurodegeneration prevented by lentiviral vector delivery of GDNF in primate models of Parkinson's disease. Science, 2000, 290: 767-773
3 Gill S S, Patel N K, Hotton G R, et al. Direct brain infusion of glial cell line-derived neurotrophic factor in Parkinson disease. Nat Med, 2003, 9: 589-595

4 Svendsen C N, Clarke D J, Rosser A E, et al. Survival and differentiation of rat and human epidermal growth factor-responsive precursor cells following grafting into the lesioned adult central nervous system. Exp Neurol,1996, 137: 376-388

5 Carpenter M K, Cui X, Hu Z Y, et al. In vitro expansion of a multipotent population of human neural progenitor cells. Exp Neurol, 1999, 158: 265-278

6 Svendsen C N, CaldwellL M A, Shen J, et al. Long-term survival of human central nervous system progenitor cells transplanted into a rat model of Parkinson's disease. Exp Neurol, 1997, 148: 135-146

7 Klein S M, Behrstock S, McHugh J, et al. Neurospheres modified to produce glial cell line-derived neurotrophic factor increase the survival of transplanted dopamine neurons. J Neurosci Res, 2002, 69: 955-965

8 Klein S M, Behrstock S, McHugh J, et al. GDNF delivery using human neural progenitor cells in a rat model of ALS. Hum Gene Ther, 2005, 16: 509-521

9 Behrstock S, Ebert A, McHugh J, et al. Human neural progenitors deliver glial cell line-derived neurotrophic factor to parkinsonian rodents and aged primates. Gene Ther, 2006, 13: 379-388

10 Georgievska B, Kirik D, Bjorklund A, et al. Aberrant sprouting and downregulation of tyrosine hydroxylase in lesioned nigrostriatal dopamine neurons induced by long-lasting overexpression of glial cell line derived neurotrophic factor in the striatum by lentiviral gene transfer. Exp Neurol, 2002, 177: 461-474

11 Gossen M, Freundlieb S, Bender G, et al. Transcriptional activation by tetracyclines in mammalian cells. Science, 1995, 268: 17661769

12 Gossen M, Bujard H. Tight control of gene expression in mammalian cells by tetracycline-responsive promoters. Proc Natl Acad Sci USA, 1992, 89: 5547-5551

13 Koponen J K, Kankkonen H, Kannasto J, et al. Doxycycline-regulated lentiviral vector system with a novel reverse transactivator rtTA2 ${ }^{\mathrm{S}}$-M2 shows a tight control of gene expression in vitro and in vivo. Gene Ther, 2003, 10: 459-466

14 Zhou B Y, Ye Z H, Gao P, et al. Inducible and reversible transgene expression in human stem cells stably transduced by novel lentiviral vectors. Stem cells, 2006, 25: 779-789

15 Kirik D, Georgievska B, Bjorklund A, et al. Localized striatal delivery of GDNF as a treatment for Parkinson disease. Nat Neurosci, 2004, 7: 105-110 
16 Judith F M, Josh R F, Wolfram K H, et al. Kruppel-associated boxes are potent transcriptionalrepression domains. Proc Natl Acad Sci USA, 1994, 91: 4509-4513

17 Deuschle U, Meyer W K, Thiesen H J. Tetracycline-reversible silencing of eukaryotic promoters. Mol Cell Biol, 1995, 15: 1907-1914

18 Moosmann P, Georgiev O, Thiesen H J, et al. Silencing of RNA polymerases II and III-dependent transcription by the KRAB protein domain of KOX1, a Krüppel-type zinc finger factor. Biol Chem, 1997, 378: 669-678

19 Kafri T, van Praag H, Gage F H, et al. Lentiviral vectors: regulated gene expression. Mol Ther, 2000, 1: 516-521

20 Mark A K, Joseph C G, Luigi N. Viral vectors for gene therapy: the art of turning infectious agents into vehicles of therapeutics. Nat Med, 2001, 7: 33-40

Open Access This article is distributed under the terms of the Creative Commons Attribution License which permits any use, distribution, and reproduction in any medium, provided the original author(s) and source are credited. 